\title{
Distribution of Photoreceptor Subtypes in the Retina of Diurnal and Nocturnal Primates
}

\author{
Kenneth C. Wikler and Pasko Rakic \\ Section of Neuroanatomy, Yale University School of Medicine, New Haven, Connecticut 06510
}

\begin{abstract}
We have used antibodies specific to either the red/greenor blue-sensitive cones in order to compare their ratio and distributions to that of the rods in the retinae of 3 primate species that differ in their capacity for color vision. We have found that the monoclonal antibody CSA-1 (Johnson and Hageman, 1988) and the polyclonal antibody 4942A, specific to the red- and green-cone opsin (Lerea et al., 1989), applied to retinal whole-mounts labeled approximately $90 \%$ of all cones in the diurnal Old-World rhesus monkey (Maccaca mulatta) and all of the cones in the nocturnal New-World owl monkey (Aotus trivirgatus) and nocturnal prosimian bushbaby (Galago garnetti). The polyclonal antibody 108B, specific to the blue-cone opsin (Lerea et al., 1989), labeled about $10 \%$ of the cones across the entire surface of the rhesus monkey retina, but failed to label any cones in the retina of the 2 nocturnal species. Only the retina of the rhesus monkey possessed an all-cone foveola in which the density of cone inner segments was 17-fold greater than that in the fovea of the owl monkey or bushbaby retina. Surprisingly, the density of cones per unit area outside of the fovea was comparable in all 3 species. Rod density in the dorsal retina was elevated in all animals examined, but was 2-3 times greater in the nocturnal species than in the rhesus monkey retina. Application of the photoreceptor-class-specific antibodies may provide further insights into the evolution and development of wavelength sensitivity in the retina, as well as enhance our understanding of normal and abnormal color vision in humans.
\end{abstract}

The primate photoreceptor mosaic consists of 4 functionally distinct cell populations: the rods, which mediate scotopic vision, and 3 cone subtypes, which mediate photopic and color vision (e.g., Jacobs, 1981; Rodieck, 1988). The 3 classes of cones are distinguished by the presence of pigments that are preferentially sensitive to short (blue-sensitive cones), middle (greensensitive cones), or long (red-sensitive cones) wavelengths of light (Schnapf et al., 1987). Knowledge about the distribution of these photoreceptor subclasses in diurnal and nocturnal primates could provide insight into the development, inheritance, and evolution of wavelength sensitivity in the retina and ulti-

Received Apr. 4, 1990; revised May 31, 1990; accepted June 7, 1990.

This research was supported by U.S. Public Health Service grant EY 02593 (P.R.) and Postdoctoral Fellowship NS 0859-01 (K.C.W.). We are grateful to Jim Hurley, Iincoln Johnson, and Connie Lerea for generously providing the antibodies, Richard Cameron for advice and useful suggestions, and Todd Preuss and Michael Schwartz for comments on the manuscript.

Correspondence should be addressed to Dr. Kenneth C. Wikler, Section of Neuroanatomy, Yale University School of Medicine, New Haven, CT 06510.

Copyright (C) 1990 Society for Neuroscience $0270-6474 / 90 / 103390-12 \$ 03.00 / 0$ mately enhance our understanding of normal and abnormal color vision in humans (c.g., Greenstein et al., 1989).

The relationship between color vision and the photoreceptor mosaic has been studied most frequently in Old-World macaque monkeys (Daw, 1984). These are diurnal species that have a distinct fovea and a topographic distribution of rods and cones that is similar to that of humans (e.g., Jacobs, 1981, 1986; Curcio et al., 1987, 1990; Packer et al., 1989; Wikler et al., 1990). In contrast, our knowledge about photoreceptors and color vision in New-World monkeys and prosimians is fragmentary. These species offer the opportunity to compare the retinae of primates that differ in their visual capacity or phyletic relatedness to either macaques or humans. For example, the owl monkey, which is the only living nocturnal anthropoid, has poorly developed color vision that is similar to that of protanomalous humans (Jacobs, 1977 a,b). There are only a few reports concerning color vision in nocturnal prosimians (Dartnall et al., 1965; Casagrande and DeBruyn, 1982). Thesc spccies are of considerable interest because they are an evolutionarily conservative group and may retain many characteristics of ancestors that are also common to monkeys, apes, and humans (Allman, 1978; Blakeslee and Jacobs, 1985).

Traditionally, studies of the number and distribution of photoreceptors in macaques have relied on morphological distinctions such as the position of nuclei and the length, width, and shape of the outer and inner segments (Ahnelt et al., 1987). However, studies of photoreceptor distribution in nocturnal anthropoids and prosimians have proved difficult because commonly used morphological criteria cannot reliably distinguish between photoreceptor subtypes.

In the past decade, immunologic markers have been developed that can distinguish between different photoreceptor subtypes. Initial antisera could only discriminate between rods and concs (Barnstable, 1980, 1987; Balkema and Drager, 1985; Akagawa and Barnstable, 1986; Lemon, 1986). However, more recently, antibodies to different cone subtypes have been generated (e.g., Szel et al., 1988; Muller et al., 1989). In the present study, we have used several antisera to different photoreceptor subtypes to examine their distribution. One of these is the monoclonal antibody CSA-1, which was initially reported to label only membranes of cones in the pig retina (Johnson and Hageman, 1988). Further studies indicate that CSA-1 selectively labels both red- and green-sensitive cones, but not blue-sensitive cones, in the rhesus monkey (Wikler and Rakic, 1989) and pig (Rohlich et al., 1989) retina. We have also used antisera raised against synthetic peptides generated from amino acid sequences for cDNAs of human photopigment polypeptides (Nathans et al., 1986; Lerea et al., 1989). These polyclonal antibodies selectively label red- and green-sensitive cones or blue-sensitive cones in 
the human retina (Lerea et al., 1989). Our use of these antisera allow us, for the first time, to examine not only the ratio and distribution of rods versus cones, but also to assess the presence or absence of wavelength-sensitive cone subtypes.

In the present study we have sought to determine: (1) whether all cone subtypes are present in nocturnal anthropoid and prosimian species, and if so, what are their cytological characteristics, dimensions, and pattern of distribution; and (2) how the distribution of photoreceptor subtypes is related to visual capacity in these 3 species (i.e., nocturnal vs diurnal).

\section{Materials and Methods}

Tissue preparation. Eyes from 4 rhesus macaque monkeys (Macaca mulatta), 4 owl monkeys (Aotus trivirgatus), and 4 thick-tailed bushbabies (Galago garnetti) were used for this study. Animals were deeply anesthetized with ketamine and sodium pentabarbitol prior to enucleation, then killed for unrelated experiments. The retinae were dissected free from other tissue layers of the eyes, marked for orientation, and briefly fixed in $4 \%$ paraformaldehyde in $0.1 \mathrm{~m}$ phosphate buffer (Stone, $1981)$.

Immunohistochemistry. Immersion-fixed retinae were rinsed in 0.1 M phosphate buffer $(\mathrm{pH}, 7.4)$ prior to overnight incubation in 1 of 3 antisera: CSA-1, a mouse monoclonal antibody diluted 1:1000 with $0.1 \%$ Triton-X 100 in $0.1 \mathrm{M}$ phosphate buffer $(\mathrm{pH}, 7.4) ; 4942 \mathrm{~A}$, an affinity-purified rabbit polyclonal antibody against the polypeptide sequence for the green/red opsin diluted $1: 10$ with $0.1 \%$ Triton-X 100 in $0.1 \mathrm{M}$ phosphate buffer $(\mathrm{pH}, 7.4)$; or the 108B affinity-purified rabbit polyclonal antibody against the polypeptide sequence for the blue opsin diluted $1: 10$ with $0.1 \%$ Triton-X 100 in 0.1 m phosphate buffer $(\mathrm{pH}$ 7.4). Following incubation in the primary antisera at $4^{\circ} \mathrm{C}$, retinae were rinsed in $0.1 \mathrm{M}$ phoosphate buffer $(\mathrm{pH} 7.4)$ and incubated in biotinylated anti-mouse or anti-rabbit IgG for $1 \mathrm{hr}$ at $22^{\circ} \mathrm{C}$. The retinae were rinsed in several changes of phosphate buffer, then incubated in an avidinbiotin-peroxidase complex (Vectastain, Vector Lab., Burlingame, CA) for $1 \mathrm{hr}$. Retinae were then reacted using diaminobenzidine $(0.05 \% 3,3-$ diaminobenzidine $\mathrm{HCl}$ and $0.003 \% \mathrm{H}_{2} \mathrm{O}_{2}$ ). In some of the bushbaby retinae, the HRP reaction product was intensified by immersion in $0.1 \%$ osmium tetroxide for 30-50 sec. Retinae were whole-mounted on gelatinized slides with the photoreceptor layer oriented up, then coverslipped in glycerin. In 1 retina of each species, nonspecific staining was assessed by omission of the primary antisera using a restricted peripheral segment of a retinal whole-mount. No immunolabeling of photoreceptor outer or inner segments was observed in these preparations.

Data collection and sampling procedures. Differential interference contrast (DIC) optics in combination with a video-overlay system was used to count photoreceptor outer or inner segments (Curcio and Sloan, 1986; Williams and Rakic, 1988; Wikler et al., 1990). DIC optics enabled us to view different planes of the photoreceptor inner and outer segments in retinal whole-mounts (Polyak, 1953). As illustrated in Figure $1 A$, rod and cone elements in unreacted rhesus macaque retinae were easily distinguishable by their size and shape near the level of the myoid (Borwein et al., 1980). Cell counts of whole-mounted retinae were made using a $100 \times$ oil immersion objective with a final optical magnification of $2600 \times$. Cells were traced on a video monitor and tabulated automatically using a microcomputer. Rods and cones were counted across the surface of each retina using a counting frame that had an area of $400 \mu \mathrm{m}^{2}$ for rods or $4550 \mu \mathrm{m}^{2}$ for cones. Samples were made at an interval of every $0.25-0.5 \mathrm{~mm}$ in the periphery and every $0.1 \mathrm{~mm}$ toward the fovea. This counting method avoids many of the difficulties encountered in determining local photoreceptor densities (for review, see Williams and Rakic, 1988). For example, correction factors for split cells (e.g., Abercrombie, 1946) were not needed because counts were taken from uncut tissue.

The cross-sectional area of rod and cone inner segments was measured at each sampling site. All cell-size measurements obtained within individual sampling sites were $\pm 5 \%$ of the mean. Photoreceptor counts were converted into local densities by calculating the number of either rods or cones per square millimeter.

\section{Results}

\section{Rhesus monkey}

The use of DIC optics allowed clear discrimination between rod and cone outer segments in unstained and unreacted wholemounts of the macaque retinae (Fig. $1 A$ ). The diameter of cone outer and inner segments at any particular retinal eccentricity, outside of the rod-free foveola, was consistently larger than the diameter of rod outer and inner segments (Fig. $1 A$ ). This distinct difference in size between rod and cone photoreceptors in the rhesus monkey cnabled us to independently and unambiguously confirm the cellular specificity of the antibodies.

In the rhesus monkey, CSA-1 and 4942A labeled cones exclusively; we did not observe immunoreactivity in any rod profiles across the entire retinal surface (Fig. $2 A, B$ ). However, in addition to an absence of labeled rods, we observed a regular and periodic absence of CSA-1 or 4942A labeling in a small proportion of profiles across the photoreceptor mosaic whose large outer segments clearly identified them as a subpopulation of cones (Fig. $2 A, B$ ). An average of $10 \%$ of all cones were unlabeled in the entire retina, and this value varied between about $5 \%$ and $15 \%$ at different eccentricities. Additionally, the unlabeled cones were distributed in a regular pattern among labeled cones.

Analysis of the distribution of CSA-1 and 4942A immunoreactivity revealed that, though the topography of labeled cones was cssentially identical for both antibodies, the localization of the reaction product within cones differed for these antisera. Immunoreactivity to the $4942 \mathrm{~A}$ antibody was restricted to the cone outer segments. In contrast, the HRP reaction product in CSA-1-labeled retinae was found not only in cone outer segments, but in their inner segments as well.

Quantitative analysis of the topographic distribution of CSA-1 or $4942 \mathrm{~A}$ immunolabeled cone profiles was similar to the distribution of the total cone population in macaque retinae determined by using DIC optics on unstained tissue (Figs. 3, 4, upper plots; Packer et al., 1989; Wikler et al., 1990). The similarity is due to the fact that only $10 \%$ of all cones were left unlabled by either $4942 \mathrm{~A}$ or CSA-1 in these immunocytochemical preparations. For example, the mean peak density of immunolabeled red/green-sensitive cones was 130,000 cones $/ \mathrm{mm}^{2}$ at the foveola and decreased more than 2-fold to 25,000 cones/ $\mathrm{mm}^{2}$ within $100 \mu \mathrm{m}$ from this peak (Figs. 3, 4, upper plots). These values are within the range of cone densities reported in previous studies (Curcio et al., 1987; Schein, 1988; Wassle et al., 1989). From this region, there was a further 10 -fold decrease in cone density to the periphery $\left(2000\right.$ cones $\left./ \mathrm{mm}^{2}\right)$. In the rhesus macaque retina, the distribution of all cones (Fig. $5 \mathrm{~A}$; Perry and Cowey, 1985; Packer et al., 1989; Wikler et al., 1990), red/greensensitive cones (Figs. 3, 4, upper plots), and ganglion cells (Perry and Cowey, 1985) is elevated in the nasal quadrant. Cone crosssectional area was smallest in the foveola at $2.2 \mu \mathrm{m}^{2}$ and enlarged to as much as $30 \mu \mathrm{m}^{2}$ in the periphery. Peak rod density in the rhesus monkey retina averaged $180,000 \mathrm{rods} / \mathrm{mm}^{2}$ and was found 3-4 $\mathrm{mm}$ dorsal to the fovea at the dorsal rod peak (DRP;

Figure 1. Photomicrographs of photoreceptor mosaics. A, Macaca mulatta. B, Aotus trivirgatus. C, Galago garnetti. DIC optics in combination with a video-overlay system were used. Arrows indicate cones. Magnification of the mosaic $(2300 \times)$ is identical for all species, illustrating the relative larger size of photoreceptors in the macaque retina and very high packing of rods in the nocturnal primates. 


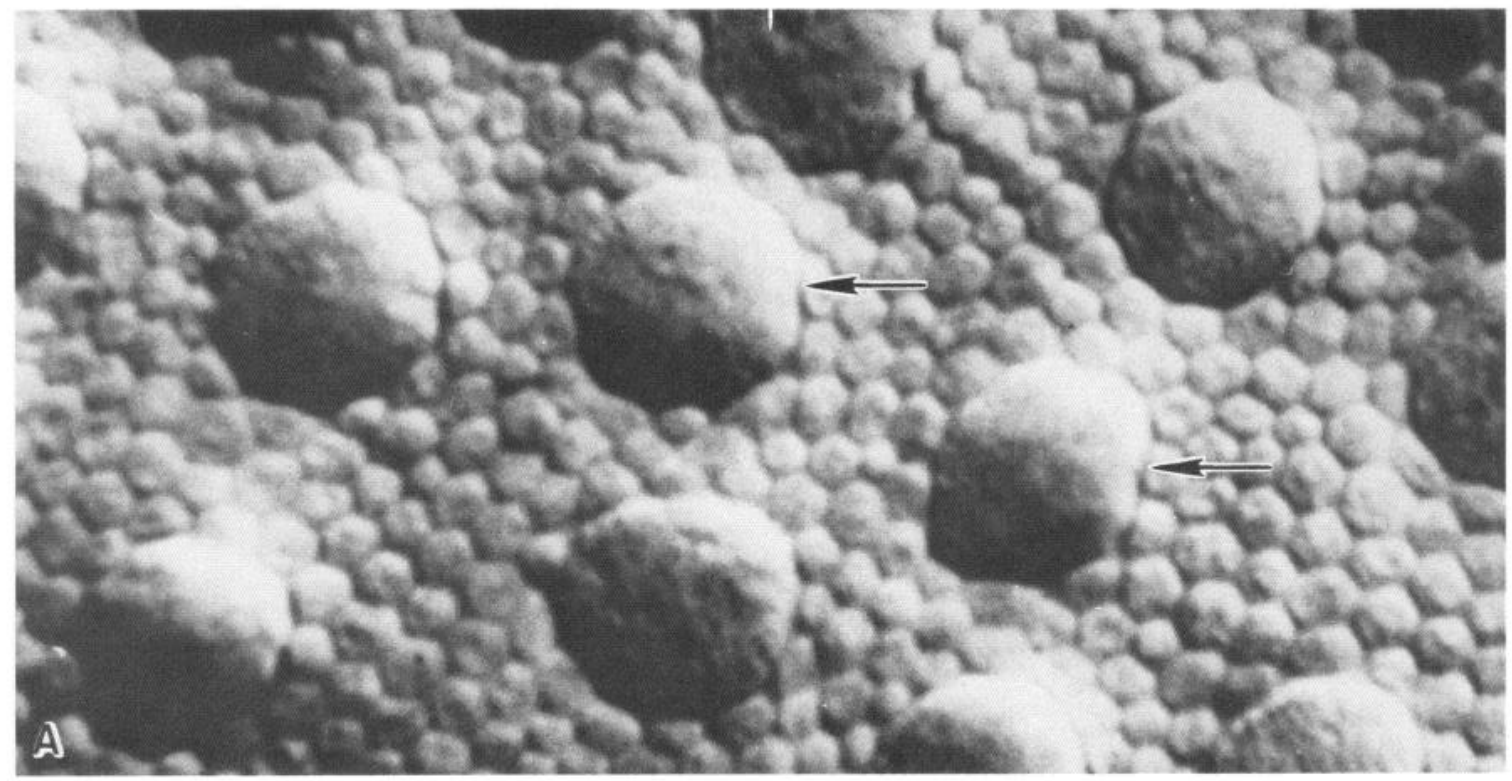

\begin{tabular}{ll}
\hline \\
$F$
\end{tabular}

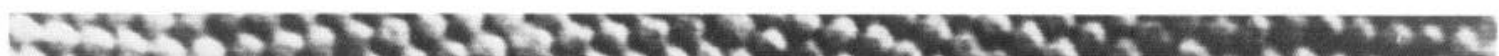

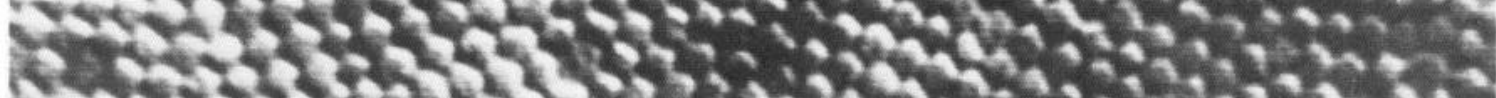

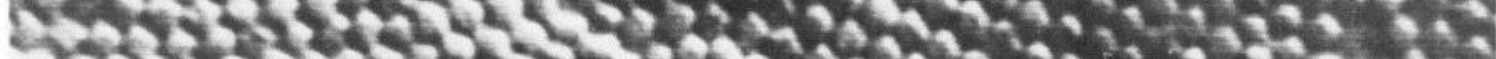

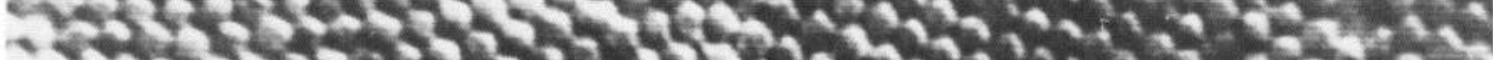

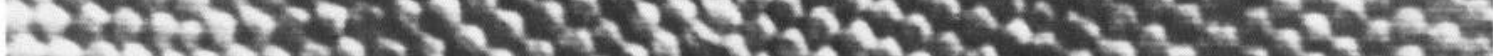

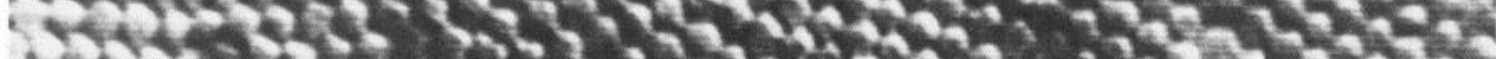

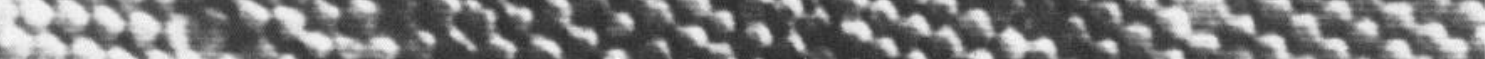

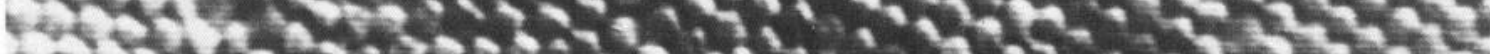

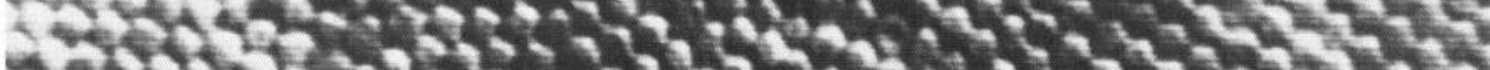
Q

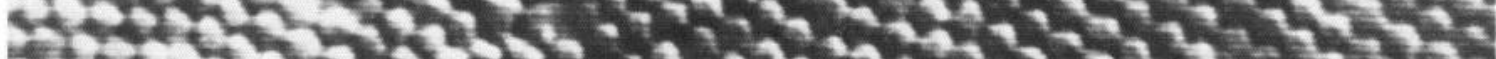

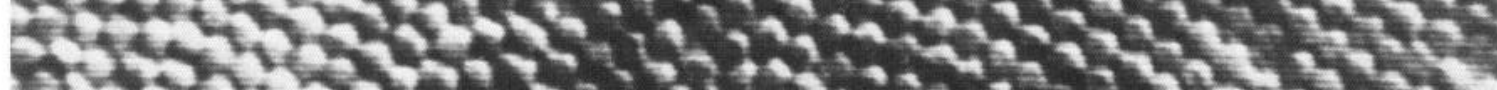

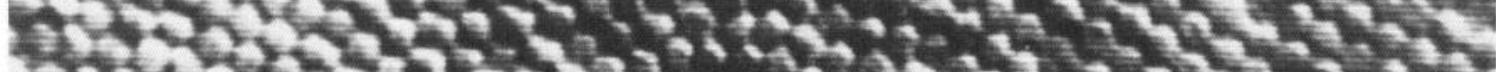

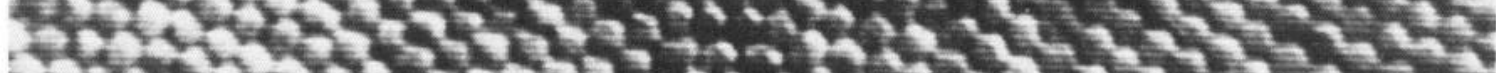

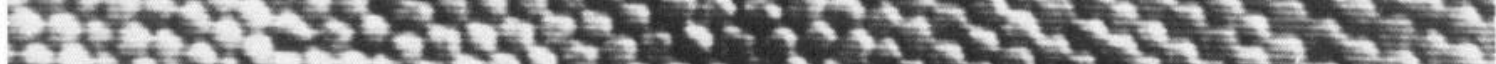
Fit

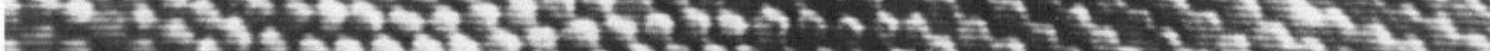

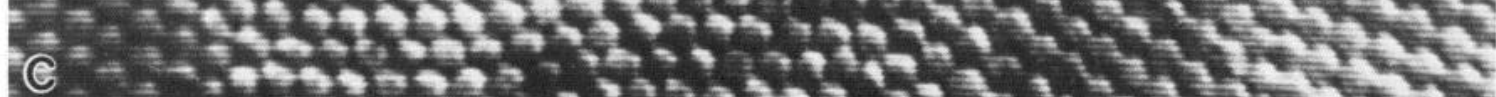



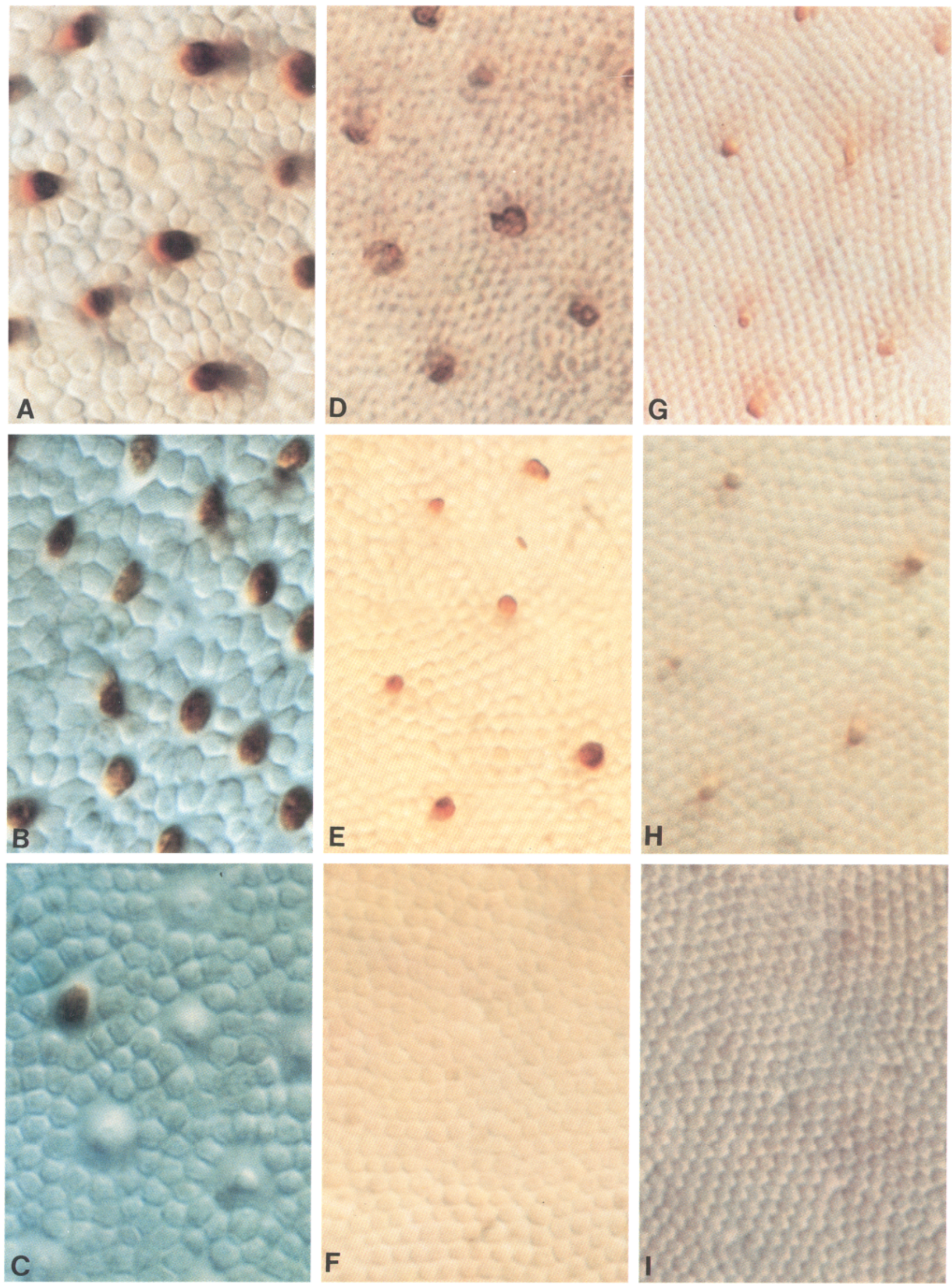

Figure 2. Photomicrographs of CSA-1-labeled $(A, D, G)$ and 4942A-labeled $(B, E, H)$ red/green-sensitive cones and 108B-labeled blue-sensitive cones $(C, F, I)$ in rhesus monkey (first column), owl monkey (middle column), and bushbaby retina (third column). Note the regular and periodic absence of labeling of putative blue-sensitive cones in the CSA-1- and 4942A-labeled rhesus monkey retinae. Magnification: $1900 \times$ for $E, F, H$, and $I ; 1350 \times$ for $A, B, C, D$, and $G$. 
Figure 3. Three-dimensional maps of the distribution of 4942A-labeled red/ green-sensitive cones (upper plot) and 108B-labeled blue-sensitive cones (lower plot) in the retina of rhesus monkey. Labeled axes indicate the density of photoreceptors $\times 1000$. N, nasal; $\mathrm{T}$, temporal; D, dorsal; V, ventral. Although there is a central elevation in the density of the blue-sensitive cones, their topography is more similar to the distribution of red/green-sensitive cones in the retinae of the nocturnal species (see Figs. 5, 6), than to red/green-sensitive cones in the retina of the rhesus monkey.

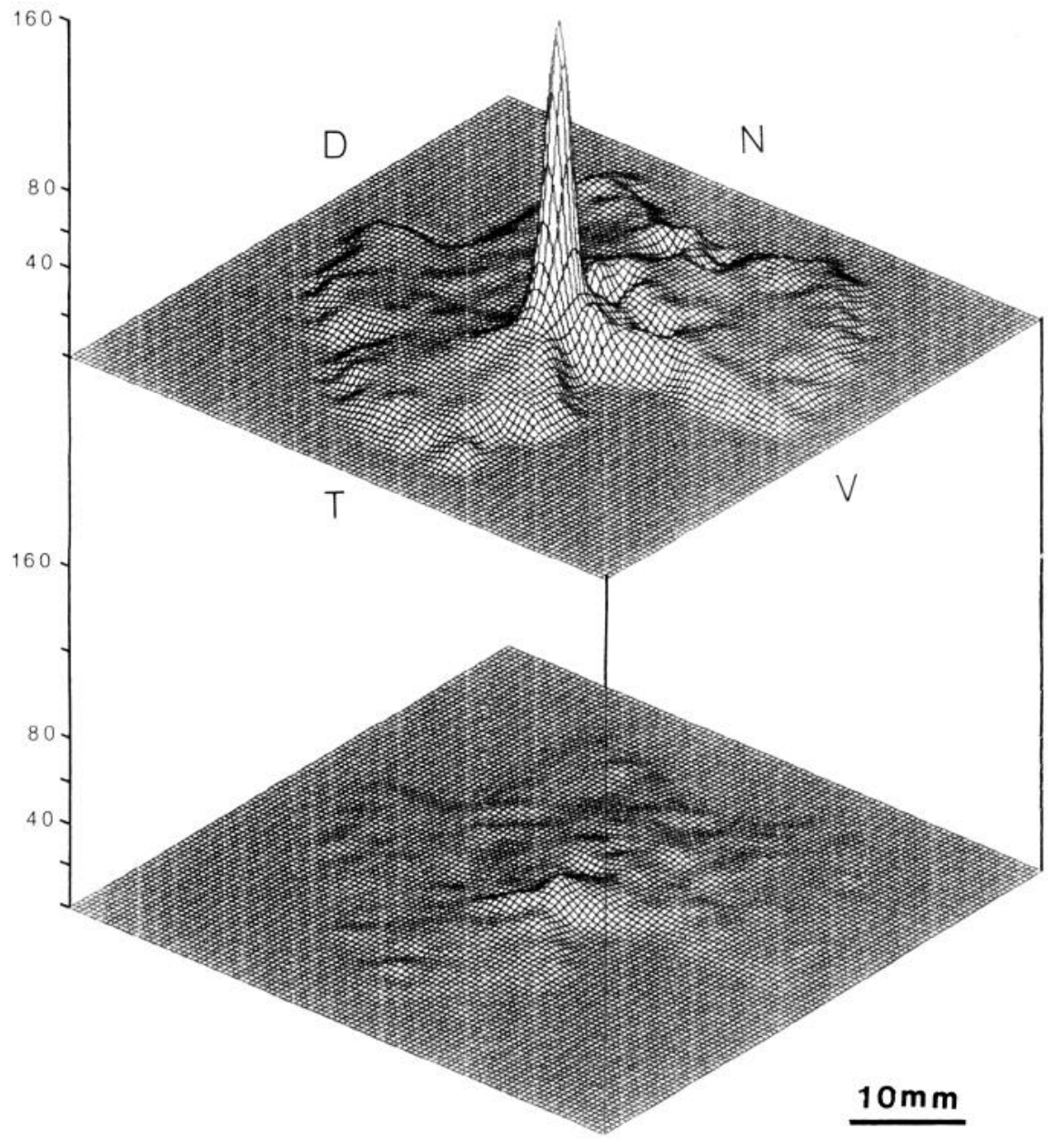

Owl monkey

The size difference between cones and rods is much less pronounced in the owl monkey than in the rhesus monkey. Nevertheless, photoreceptor cell types in whole-mounted owl monkey retinae could be readily identified using DIC optics (Fig. 1B). Both the CSA-1 and 4942A antibodies labeled cones exclusively (Fig. 2D,E). However, in contrast to the results obtained for the rhesus monkey, both antibodies labeled all cone cell profiles. Every profile, independently identified as a cone on the basis of its relatively larger inner segment, was immunolabeled by the 4942A antibody. Comparison of immunolabeling in the owl and rhesus monkey retina showed that a similar distribution of labeled cones existed outside of the fovea (Fig. 2A,D and $B, E$ ).

The density of red-green-sensitive cones was quantified across the retinal surface. We found neither a foveola or a major central peak in cone density in the owl monkey. The highest plateau in the density of immunocytochemically labeled cones was in central retina and ranged between 6600 and 7000 cones/ $\mathrm{mm}^{2}$. This value decreased to approximately 2800 cones $/ \mathrm{mm}^{2}$ in the periphery (Figs. $5 \mathrm{~B}, 6 \mathrm{~B}$ ). Thus, unlike in the rhesus monkey, the central to peripheral gradient in cone density in the owl monkey was only 2-3-fold.

In retinal whole-mounts of the owl monkey, the region of the foveal pit or area centralis can be located with the aid of a

dissection scope and was found in an avascular area temporal that the rate of change in density of this cone subtype was more gradual than that determined for the red/green-sensitive cones. 

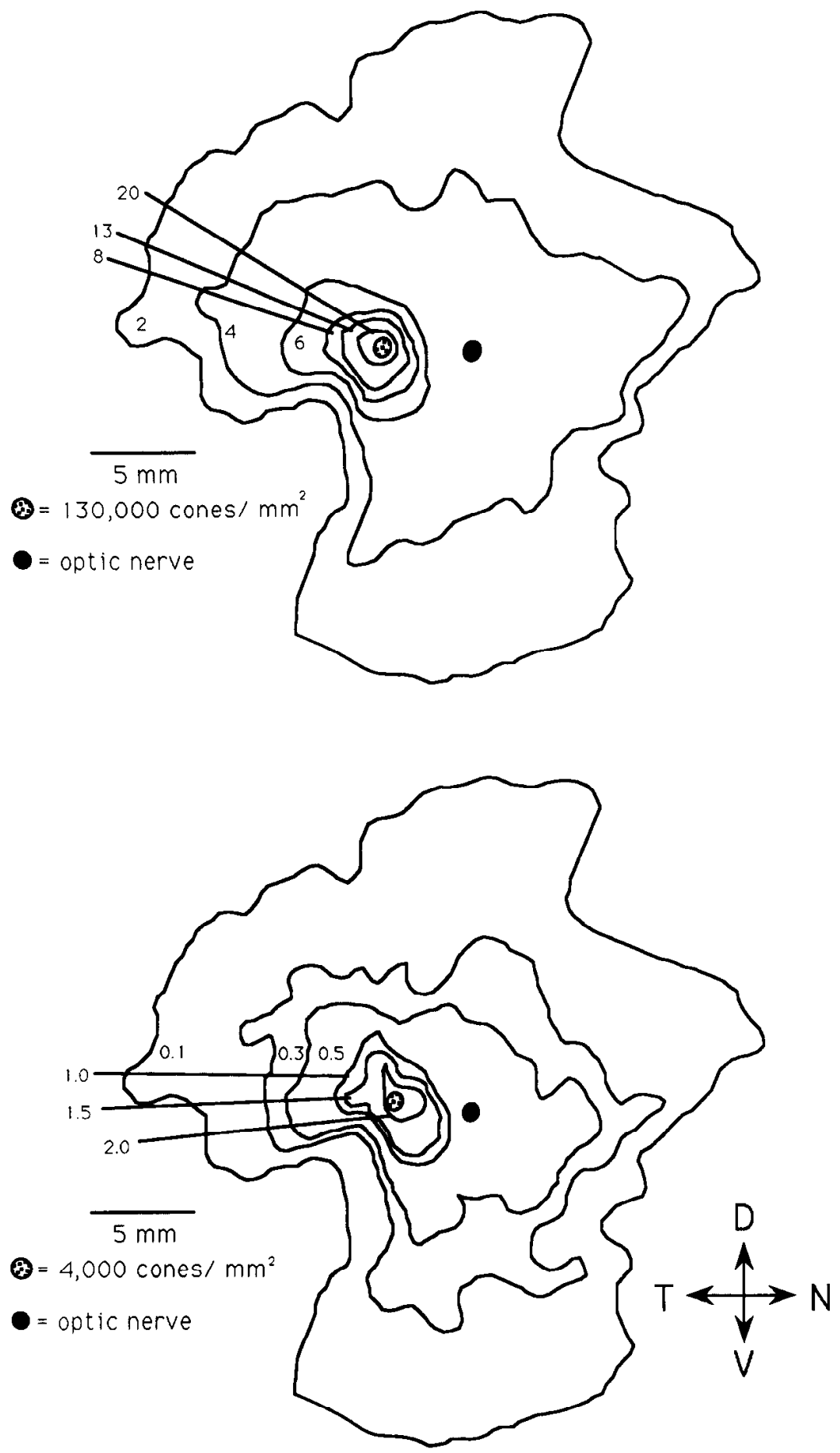

Figure 4. Isodensity contour plots of the distribution of 4942A-labeled red/ green-sensitive cones (upper plot) and 108B-labeled blue-sensitive cones (lower plot ) in the retina of the rhesus monkey, as illustrated in Figure 3. Numerical values indicate the density of photoreceptors $\times 1000$. N, nasal; $T$, temporal; $\mathrm{D}$, dorsal; $\mathrm{V}$, ventral.

to the optic disk. However, as illustrated in the isodensity contour plot in Figure 6, peak cone density was not localized in the foveal region in this species. Cone density at the fovea ranged between 5000 and 6000 cones $/ \mathrm{mm}^{2}$, approximately 1000 cones/ $\mathrm{mm}^{2}$ less dense than the peak, which was located about $2 \mathrm{~mm}$ dorsal to the fovea.

We detected no evidence of 108B-immunoreactive cones in owl monkey retinae (Fig. $2 F$ ). The use of DIC optics combined with video microscopy enabled us to discriminate between cones and rods on the basis of the larger size of the cone inner segments (Fig. $1 \mathrm{~B}$ ). In contrast to the regular distribution of 108B-labeled cones observed in the rhesus monkey retina, thorough exami- nation of the owl monkey photoreceptor mosaic $(n=2)$ failed to reveal a single $108 \mathrm{~B}-$ labeled cone profile.

The ratio of rods to cones in the owl monkey was approximately 50:1 over much of the retinal surface. However, ratios between these 2 photoreceptor classes varied significantly with change in eccentricity. In the far periphery of the dorsal retina, the rod-to-cone ratio reached 93:1. However, at the level of the fovea, it dropped dramatically to $14: 1$. Because the density of cones decreased only 2-3-fold from the central to the peripheral retina, the changing numerical proportion of cones to rods reflects significant regional variation in the local density and size of rods across the retinal surface. 

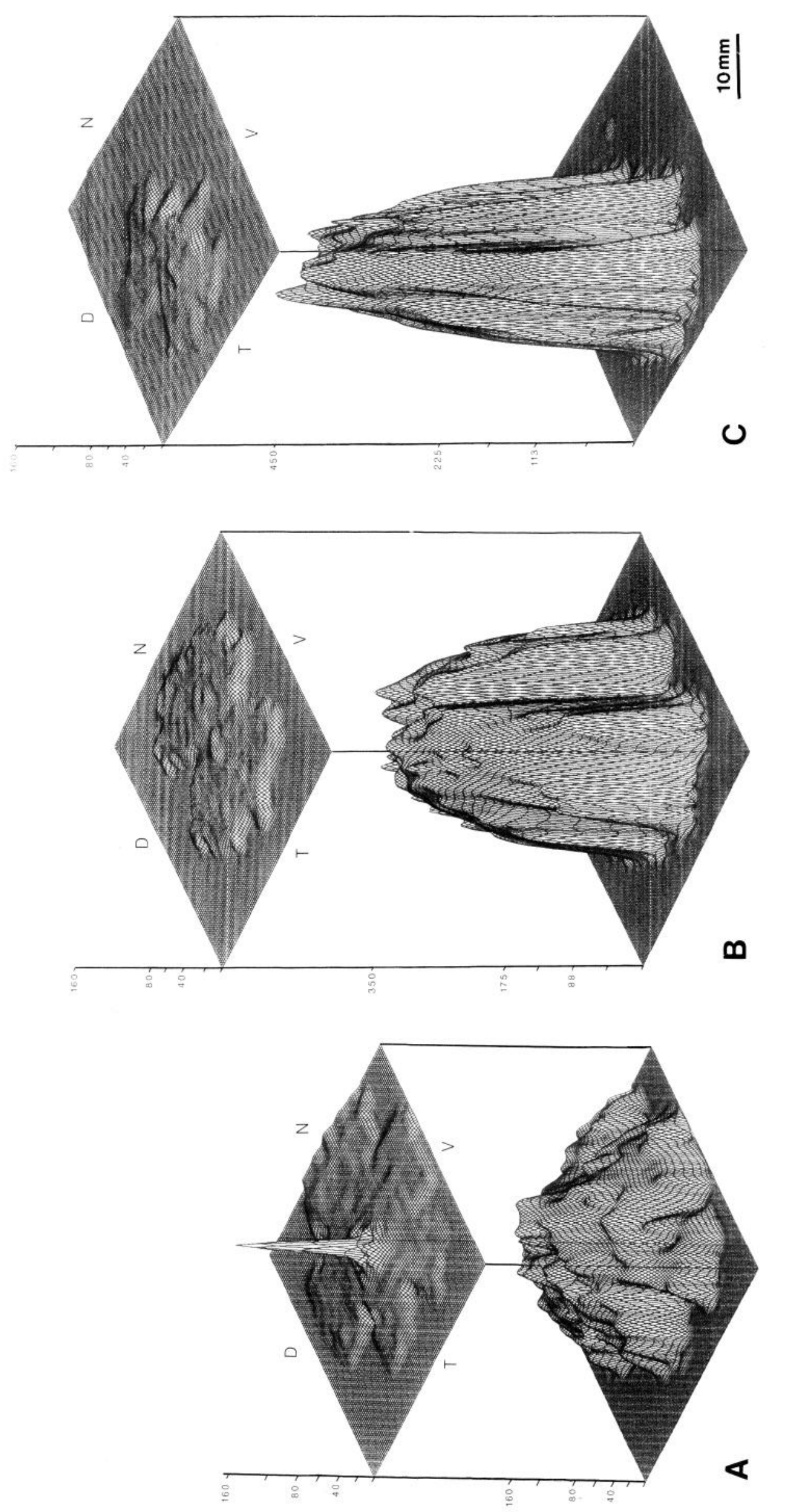

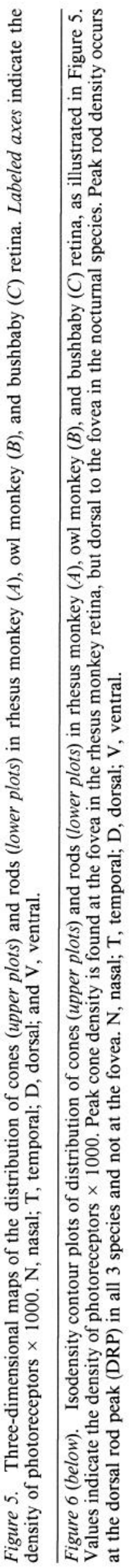




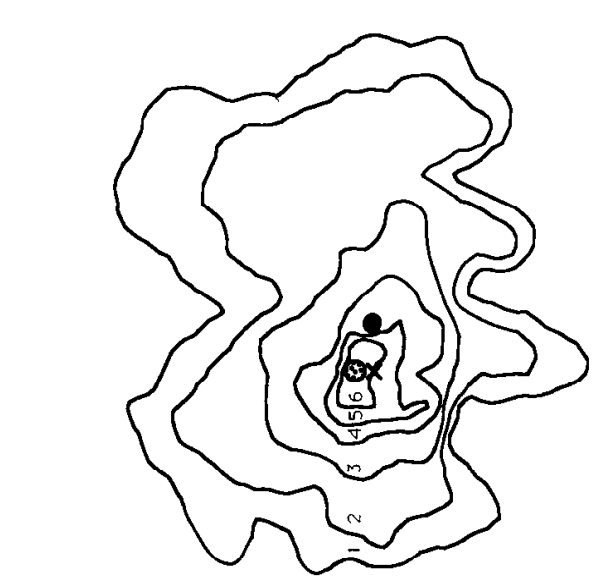

0
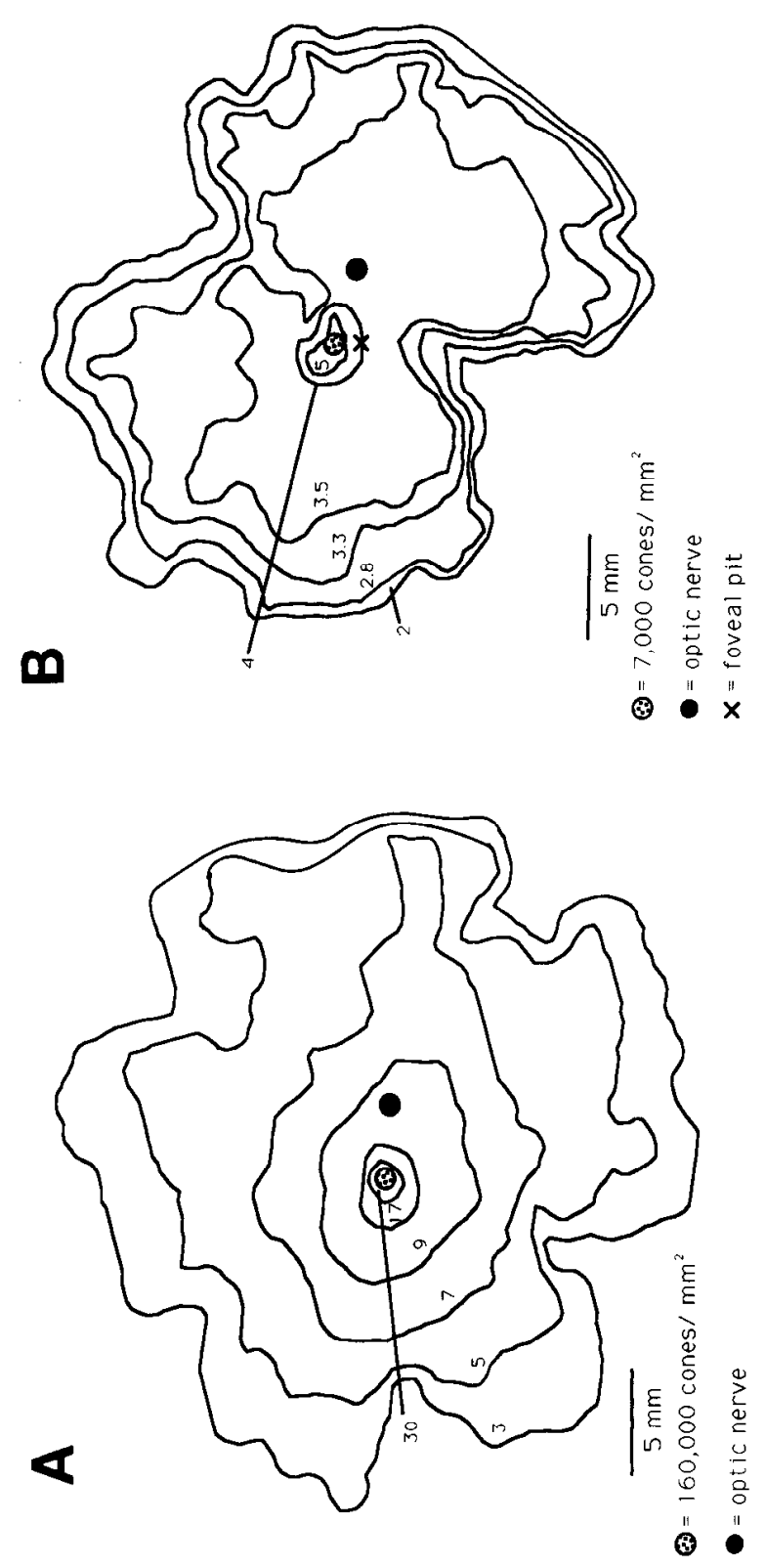
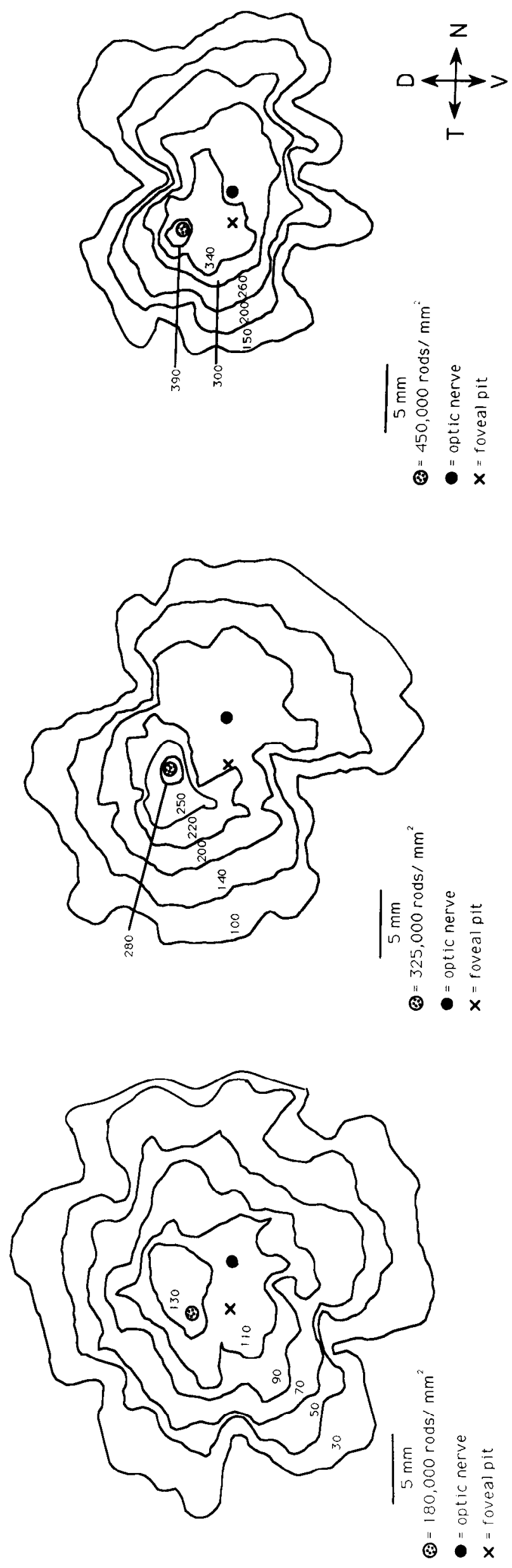


\section{Thick-tailed bushbaby}

Using video-enhanced DIC optics to view unreacted and unstained retinae in this species (Fig. 1C), it was difficult to discern between photoreceptor subtypes employing routine morphological criteria to discriminate between rods and cones. Application of either 4942A or CSA-1 antibodies to whole-mounted retinae of the nocturnal bushbaby revealed an extensive and regular array of immunoreactive outer segments (Fig. $2 G, H$ ). Because the diameter of all photoreceptors in the bushbaby retina was significantly smaller than that of homologous cell types in the rhesus monkey (cf. Fig. 1A,C), discrimination between rods and cones on the basis of the area of outer or inner segments alone was extremely difficult.

Detailed measurements using DIC optics at high magnification revealed that immunolabeled photoreceptors had outer and inner segments that were consistently larger than that of surrounding nonlabeled receptors. For example, in the parafoveal region of the bushbaby retina, the average area of labeled outer segments was greater than the surrounding unlabeled outer segments by about $50 \%\left(1.5 \mu \mathrm{m}^{2}\right.$ vs $\left.1.0 \mu \mathrm{m}^{2}\right)$. Furthermore, immunolabeled inner segments were larger than unlabeled inner segments by approximately the same percentage. Finally, the combined length of the labeled inner and outer segment was about $40 \mu \mathrm{m}$, whereas the combined inner and outer segment length of unlabeled profiles was approximately $60 \mu \mathrm{m}$. Thus, our analysis reveals that CSA-1 and $4942 \mathrm{~A}$ labeled a population of receptors that possessed inner and outer segments that consistently showed a larger diameter and shorter length than the inner and outer segments of surrounding label-free receptor profiles.

Immunolabeled retinal whole-mounts provided an ideal preparation for precisely determining the distribution of cones in the bushbaby (e.g., Figs. $2 G, H, 5 C, 6 C$ ). The density of labeled cones in the bushbaby, like the other species examined in this study, was sampled at the fovea, as well as across the retinal surface. The immunoreactive outer segments were distributed regularly and relatively evenly over the surface of the retina. Although the foveal pit or area centralis in the bushbaby was not as pronounced as that in Old- or New-World monkeys, a well-defined avascular area located temporal to the optic disk (DeBruyn et al., 1980; Stone and Johnson, 1981) could be located and can be considered the foveal region in this species. The existence of a foveola with a high density of cones has not been reported in previous studies (Ogden, 1975; DeBruyn et al., 1980). However, the region of the fovea, as defined by the vascular pattern of the retina (Stone and Johnson, 1981), could bc identified.

The density of CSA-1- or 4942A-labeled photoreceptors in the region of the fovea averaged between 5700 and 6000 cones/ $\mathrm{mm}^{2}$. However, unlike macaques and humans, the foveal region in the bushbaby did not coincide with the location of the peak cone density. Peak cone density in the bushbaby, as revealed by the immunostaining observed with the $4942 \mathrm{~A}$ or CSA-1 antibodies, ranged between 7500 and 8500 cones $/ \mathrm{mm}^{2}$ and was located approximately $1 \mathrm{~mm}$ dorsal to the fovea (Figs. $5 \mathrm{C}, 6 \mathrm{C}$, upper plots). Cone outer segments had an area of $1.5 \mu \mathrm{m}^{2}$ at the fovea and approached $1 \mu \mathrm{m}^{2}$ in the region of peak density. In pericentral retina, cone density ranged from 4000 to 5000 cones/ $\mathrm{mm}^{2}$ and decreased in the retinal periphery to about 1500 cones/ $\mathrm{mm}^{2}$. Thus, in the bushbaby retina, the central-to-peripheral gradient in cone density was little more than 2-3-fold.
Examination of 3 immunoreacted bushbaby retinae incubated with the 108B antibody failed to reveal any positively labeled cone outer segments (Fig. 2I). Although it was difficult to differentiate cones from rods in the bushbaby retina, at high magnification it was at times possible to identify periodic pits or depressions in the outer surface of the mosaic that appeared to correspond in frequency to CSA-1- or 4942A-immunoreactive profiles (cf. Fig. $2 G, H$ to $I$ ). Our examination of these profiles across the retinal surface failed to localize any 108B-immunoreactive receptors in the bushbaby retina.

Immunolabeling with the CSA-1 and 4942A antibodies enabled us to quantify the ratio of rods to cones in the bushbaby retina. Our analysis showed that there are at least 60 rods for every cone over much of the retinal surface, with values ranging as high as 100:1 and as low as 39:1. The higher proportion of rods to cones was found in the extreme retinal periphery, whereas the lowest ratio was located at the fovea. The density of rods was very high across the entire surface of the bushbaby retina, but decreased abruptly at the fovea. However, within $0.5-1 \mathrm{~mm}$ from the fovea, the ratio of rods to cones increased to $60: 1$.

\section{Discussion}

The availability of the cone-specific monoclonal antibody CSA1 , as well as 2 antibodies raised against defined polypeptide sequences for either the red/green opsin (4942A) or the blue opsin (108B), allowed us to identify subpopulations of cones across primate species with different color vision capacities. Our results with the CSA- 1 and 4942A antisera reveal that red- and green-sensitive cones are present in all species examined. In contrast, the results with the $108 \mathrm{~B}$ antibody suggest that bluesensitive cones are present in diurnal Old-World monkeys but are missing in the retinae of nocturnal New-World monkeys and prosimians. Examination of immunolabeled retinae in this study provides the first direct evidence that the red/green-sensitive cone pigment is present in nocturnal prosimian species. Furthermore, these immunocytochemically identified cones are organized in a regular array across the retina. The distribution of labeled cones and the proportion of cones to rods show some surprising similarities and differences between primate species that may explain associated differences in visual capacity.

\section{Immunolabeled cone subtypes in the rhesus monkey}

Double-labeling experiments have demonstrated that the CSA-1 and 4942A antibodies specifically label both red- and greensensitive cones in the pig (Rohlich et al., 1989) and human (Lerea et al., 1989) retina. The antibody 108B labels only bluesensitive concs in the human retina, and the distribution is reciprocal to that of 4942A-labeled cones (Lerea et al., 1989).

As expected, in the present study, these 3 antibodies labeled discrete subtypes of cones in the rhesus monkey retina. Using morphological criteria, we were able to determine that all immunolabeled photoreceptor profiles in the macaque retina were cones. Rods were never immunolabeled with any of the 3 antibodies. Careful examination of CSA-1- or 4942A-immunolabeled macaque retinae consistently revealed that an average of about $10 \%$ of the cone outer segments were not immunoreactive. The proportion and relative distribution of cones in the macaque retina labeled with the blue-opsin-specific antibody $108 \mathrm{~B}$ appears to correspond to the distribution of cone outer segments left unlabeled by CSA-1 or $4942 \mathrm{~A}$ antibodies. Furthermore, the pattern of labeling of cone subtypes with the set of antibodies used in this study is similar to the distribution of 
cone subtypes in the rhesus monkey retina identified by other means (Marc and Sperling, 1977; de Monasterio et al., 1981, 1985; Szel et al., 1988).

\section{Cone subtypes of the nocturnal New-World monkey and prosimian}

Using the distribution of the photoreceptor subtypes of the macaque retina as a point of comparison, we applied these antibodies to the retinae of nocturnal primates. This comparison is of particular interest because it has been hypothesized that differences in color vision may be correlated with differences in the presence or distribution of photoreceptor subtypes (Walls, 1942; Ehrich and Calvin, 1967; Hughes, 1977; DeValois and Jacobs, 1984). For example, behavioral studies indicate that, though the owl monkey can discriminate between red and green light (Ehrich and Calvin, 1967), its photopic spectral sensitivity is low and resembles protanomalous trichromacy in humans (Jacobs, 1977a, 1981). Additionally, though diurnal prosimians (e.g., ring-tailed lemurs) are known to be trichromats (Blakeslee and Jacobs, 1985), there is no comparable study of color vision in nocturnal prosimians. However, in none of these nocturnal primate species has the proportion and relative distribution of the different photoreceptor subtypes been examined.

Ogden was able to histologically demonstrate the presence of cones in the owl monkey retina and, after quantification of a limited sampling area, concluded that the retina of this primate species is rod dominated (Ogden, 1975). Having demonstrated that CSA-1 and 4942A label both red-and green-sensitive cones and that 108B labels blue-sensitive cones in Old-World diurnal monkeys, we also examined the pattern of immunoreactivity in nocturnal prosimians and New-World monkeys. Our study clearly demonstrates the presence of immunoreactive cones at all eccentricities in the retinae of both the bushbaby and the owl monkey. Unlike diurnal macaques, all cones in our nocturnal primates were labeled with the CSA-1 and 4942A antibodies. Conversely, the 108B antibody failed to label any cone profiles in either the owl monkey or the bushbaby retina.

One explanation for our inability to find blue-sensitive cones in the nocturnal species could be that the $108 \mathrm{~B}$ antibody recognizes an epitope of the blue-sensitive cone pigment that is not maintained across these primates. This finding could be interpreted as indicating that this epitope has not been conserved through primate evolution. However, several lines of evidence lead us to suggest that blue-sensitive cones may not be present in the retina of owl monkeys and bushbabies. First, our examination of CSA-1 - or 4942A-reacted retinae indicated that all cones in the retinae of the nocturnal species are immunoreactive and thus are red/green-sensitive. It is unlikely, moreover, that blue-sensitive cones in the nocturnal species are reduced in size and thus are indistinguishable from rods, because previous studies have clearly demonstrated that all cones in the owl monkey are consistently larger in size than adjacent rods (Ogden, 1975). Second, we recently have labeled blue-sensitive cones in the retinae of the squirrel monkey (Saimiri sciureus) and the opossum (Monodelphis domestica) using the 108B antibody (unpublished observations). Because this antibody successfully labels an epitope of the blue-cone opsin in species as diverse as humans and marsupials, we would expect that the 108B antibody should label cones in the nocturnal primates as well, if they were present. Finally, microspectrophotometry of photoreceptors in the retina of the squirrel monkey, a diurnal New-World monkey, indicated that only $4 \%$ of the total pop- ulation of cones were short-wavelength sensitive in this species (Bowmaker et al., 1985). By extension, one could expect that the owl monkey, a nocturnal New-World species, may possess an even lower number of blue-sensitive cones, or that they may be absent.

These data raise the possibility that, though classified as trichromats, the color-vision abilities of these nocturnal species may not be subserved by 3 subpopulations of cones as is the case for Old-World monkeys and humans. Instead, in the owl monkey and bushbaby, trichromatic color vision may be subserved by only 2 cone pigments and rhodopsin (Blakeslee and Jacobs, 1985). We cannot directly address this possibility in the present study because, due to the $96 \%$ sequence homology between the red and green photopigments, these wavelength-sensitive pigments presently cannot be differentiated with available antisera.

\section{Cone and rod topography in nocturnal and diurnal primates}

In addition to the obvious difference in the frequency of cone subtypes for the species examined in this study, 2 important features of photoreceptor topography differed between diurnal primates with well-developed color vision abilities and species that are active at twilight or at night.

First, in the diurnal Old-World macaques, there was a high density of cones in the fovea, which was not found in the nocturnal New-World or prosimian species (see Figs. 5, 6). In fact, peak cone density was not located at the fovea (as defined by either the extent of the pit or the vascular pattern of the retina) in both nocturnal species. Therefore, the peak of the cone topography is not necessarily centered as the foveal pit in all primates. Evidently, only in Old-World higher primates such as rhesus monkeys and in humans has the foveal region become specialized for acuity and color vision. This specialization occurs by the clearing of cells in the ganglion and inner nuclear layers (Hendrickson and Kupfer, 1976) and cone pedicles in the outer plexiform layer (Schein, 1988), as well as by the development of a cone peak in spatial register with the foveal pit (Curcio et al., 1987). Recent experimental manipulations of the primate retinal ganglion cell layer indicate that the development of a foveal pit is related to ganglion cell density (Leventhal et al., 1989). This finding, in addition to the results in the present study, indicate that the development of the primate fovea may be independent of the development of peak cone density at the foveola.

Second, we found a much higher ratio of rods to cones in the owl monkey and bushbaby, and a much higher density of rods, in comparison to the rhesus monkey retina. This high rod density is the result of their smaller diameter in the nocturnal species (see Fig. 1). The highest rod-to-cone ratio in the rhesus monkey was $35-40: 1$, with the highest density of rods averaging 180,000 rods $/ \mathrm{mm}^{2}$ in the dorsal retina. In contrast, the highest proportion of rods to cones was 93:1 in the owl monkey and 100:1 in the bushbaby. Rod peak density was $325,000 \mathrm{rods} / \mathrm{mm}^{2}$ in the owl monkey retina and $450,000 \mathrm{rods} / \mathrm{mm}^{2}$ in the bushbaby retina (Figs. 5, 6).

The difference in rod density between the 2 nocturnal species suggests that the overall proportion of photopic to scotopic receptors in these primates may vary not only according to a species adaptation to visual activity at either high or low illumination levels, but also to the species' phylogenetic history. We suggest that the lower density of rods found in Aotus, in contrast to that in the bushbaby retina, may reflect the owl 
monkey's diurnal ancestry (Ciochon and Fleagle, 1985; Pariente, 1979).

One of the features of the photoreceptor mosaic shared among the 3 species examined in this report is the density of red/greensensitive cones. In the diurnal macaque, outside of the fovea, the density of these cones was similar to that across the entire retina in the nocturnal prosimian and New-World monkey (Figs. $5,6)$. Due to the smaller size of the owl monkey and bushbaby retina [given the comparable size of the visual field across all primates (Casagrande and DeBruyn, 1982)], the number of cones per degree of visual field is about $1 / 2$ of that in the rhesus monkey, in spite of the similar density per unit of surface area. However, the major difference in cone topography between diurnal and nocturnal primates is a restricted $1-2-\mathrm{mm}^{2}$ area in the central rctina of Old-World monkeys and humans.

Finally, in all 3 species examined, the peak in rod density was located in dorsal retina (the DRP) and not within or near the fovea (see isodensity contour plots in Fig. 6). Although expected for the diurnal species, we were surprised to find the scotopic peak not aligned with the fovea in nocturnal primates. The decrease in rod density and increase in their size at the fovea in nocturnal primates suggests that the foveal region in these species may be important for sensitivity at low light levels and not for spatial acuity.

\section{Issues concerning the phylogeny of primate color vision}

The nocturnal prosimians are considered to be the most primitive living primates and resemble, in a number of anatomical and behavioral respects, the ancestral species that gave rise to present-day monkeys, apes, and humans (e.g., Allman, 1981). Cranial endocasts indicate that Eocene primatcs had large cycs specialized for night vision and a relatively large primary visual cortex in the occipital lobe, much like the present-day bushbaby (Martin, 1989). Our comparison of the retinae of living nocturnal and diurnal primates appears to buttress the long-held view that early primates were nocturnal. The relatively large eyes, presence of a reflective tapetum lucidum, and lack of a central elevation in cone density and relative increase in rod density, as well as the enhanced scotopic acuity (Langstan et al., 1986) found in nocturnal prosimians relative to diurnal primates, suggest that the early primate retina was specialized, in all probability, for nocturnal vision.

The most likely function of cones in primitive nocturnal primates may not have been related to color vision or photopic acuity because cone size and spacing per degree of visual field in the retina of the owl monkey and bushbaby can only support a level of photopic acuity significantly inferior to that of macaques or humans (Jacobs, 1977a). Furthermore, nocturnal primates are exposed predominantly to green and blue wavelengths rather than to the full wavelength spectrum available at high illumination levels (Wright, 1989). Because wavelength discrimination is coded by the differential output of 2 different photoreceptor types (rather than the sum of their output, as is the case for intensity discriminations), there is little information available in the environment for color discrimination at low light levels (DeValois and Jacobs, 1984).

The presence of cones in nocturnal primates and the fact that they are probably not used extensively for wavelength discrimination suggest that the original function of cones may have differed from their current function in diurnal primates. For example, the photopic system of early primates may have been important for the detection of circadian fluxes in illumination levels. Field observations of nocturnal prosimians indicate that the onset and cessation of locomotor activity corresponds to small differences in ambient light levels at dusk and dawn (Bearder and Martin, 1980). These intensity levels correspond to those necessary for the activation of cones at low light levels (Martin, 1989). Also, Jacobs et al. (1979) suggest that the visual capacity of the owl monkey "to discriminate relatively fast temporal changes in very dim light" may be related to the function of cones at low illumination levels. Further studies exploiting the use of immunocytochemical probes, which allow precise identification and unambiguous quantification of photoreceptor types in comparative and developmental studies of the visual system, may elucidate the ways in which developmental mechanisms for the generation of photoreceptor number and distribution are altered during evolution.

\section{References}

Abercrombie M (1946) Estimation of nuclear populations from microtome sections. Anat Rec 94:239-247.

Ahnelt PK, Kolb H, Pflug R (1987) Identification of a subtype of cone photoreceptor, likely to be blue sensitive in the human retina. J Comp Ncurol 255:18-34.

Akagawa K, Barnstable CJ (1986) Identification and characterization of cell types in monolayer cultures of rat retina using monoclonal antibodies. Brain Res 383:110-120.

Allman J (1978) Evolution of the visual system in the early primates. In: Progress in psychobiology and physiological psychology, Vol III (Sprague JM, Epstein AN, eds), pp 1-53. New York: Academic.

Allman J (1981) Reconstructing the evolution of the brain in primates through the use of comparative neurophysiology and neuroanatomical data. In: Data in primate brain evolution: methods and concepts. (Armstrong E, Falk D, eds), pp 13-28. New York: Academic.

Balkema GW, Drager UC (1985) Light-dependent antibody labeling of photoreceptors. Nature 316:630-633.

Barnstable CJ (1980) Monoclonal antibodies which recognize different cell types in the rat retina. Naturc 286:231-235.

Barnstable CJ (1987) Immunological studies of the diversity and development of the mammalian visual system. Immun Rev 100:47-78.

Bearder SK, Martin RD (1980) The social organization of a nocturnal primate revealed by radiotracking. In: Handbook of biotelemetry and radiotracking (Laner AM, McDonald DW, eds), pp 633-648. Oxford: Pergam.

Blakeslee B, Jacobs GH (1985) Color vision in the ring-tailed lemur (Lemur catta). Brain Behav Evol 26:154-166.

Borwein B, Borwein D, Medeiros J, McGowan JW (1980) The ultrastructure of the monkey foveal photoreceptors, with special reference to the structure, shape, and size and spacing of the foveal cones. Am J Anat 159:125-146.

Bowmaker, JK, Jacobs GH, Spegel-Walter DJ, Mallon JD (1985) Two types of trichromatic squirrel monkey share a pigment in the redgreen spectral region. Vision Res 12:1937-1946.

Casagrande VA, DeBruyn EJ (1982) The galago visual system: aspects of normal organization and developmental plasticity. In: The lesser bushbaby (galago) as an animal model: selected topics (Haines DE, ed). Boca Raton: CRC.

Ciochon RL, Fleagle JG (1985) Primate evolution and human origins. New York: A. DeGruyter.

Curcio CA, Sloan KR Ir (1986) Computer aided morphometry using video-mixed microscopic images and computer graphics. Anat Rec 214:329-337.

Curcio CA, Sloan KR Jr, Packer O, Hendrickson AE, Kalina RE (1987) Distribution of cones in human and monkey retina: individual variability and radial asymmetry. Science 236:579-582.

Curcio CA, Sloan KR, Kalina RE, Hendrickson AE (1990) Human photoreceptor topography. J Comp Neurol 292:497-523.

Dartnall HJA, Arden GB, Ikeda H, Luck PC, Rosenberg CM, Pedler $\mathrm{H}$, Tansley K (1965) Anatomical, electrophysiological, and pigmentary aspects of vision in the bush baby: an interpretive study. Vision Res 5:399-424.

Daw NW (1984) The psychology and physiology of color vision. Trends Neurosci 7:330-335. 
DeBruyn EJ, Wise VL, Casagrande VA (1980) The size and topographic arrangement of retinal ganglion cells in the galago. Vision Res 20:315-327.

de Monasterio FM, Schein SJ, McCrane EP (1981) Staining of bluesensitive cones of the macaque retina by a fluorescent dye. Science 213:1278-1280.

de Monasterio FM, McCrane EP, Newlander JK, Schein SJ (1985) Density profile of blue-sensitive cones along the horizontal meridian of macaque retina. Invest Ophthalmol Vis Sci 26:289-302.

DeValois RL, Jacobs GH (1984) Neural mechanisms of color vision. In: Handbook of physiology, Vol. III, Sensory processing (DarianSmith I, ed), pp 425-456. Washington, DC: American Physiological Society.

Ehrich A, Calvin WH (1967) Visual discrimination behavior in galago and owl monkey. Psychon Sci 9:509-510.

Greenstein VC, Hood DC, Ritch R, Steinberger D, Carr RE (1989) S (blue) cone pathway vulnerability in retinitispigmentosa, diabetes and glaucoma. Invest Ophthalmol 30:1732-1737.

Hendrickson A, Kupfer C (1976) The histogenesis of the fovea in the macaque monkey. Invest Ophthalmol 15:746-756.

Hughes A (1977) The topography of vision in mammals of contrasting life style: comparative optics and retinal organization. In: The visual system in vertebrates (Crescitelli F, ed), pp 615-755. New York: Springer-Verlag.

Jacobs GH (1977a) Visual capacities of the owl monkeys (Aotus trivirgatus). I. Spectral sensitivity and color vision. Vision Res 17:811820.

Jacobs GH (1977b) Visual capacities of the owl monkeys (Aotus trivirgatus). II. Spatial contrast sensitivity. Vision Res 17:821-825.

Jacobs GH (1981) Comparative color vision. New York: Academic.

Jacobs GH (1986) Color vision variations in non-human primates. Trends Neurosci 9:320-323.

Jacobs GH, Tootell RBH, Blakeslee B (1979) Visual capacities of the owl monkey (Aotus trivirgatus): temporal contrast sensitivity. Folia Primatol 32:193-199.

Johnson LV, Hageman GS (1988) Characterization of molecules bound by the cone photoreceptor-specific monoclonal antibody CSA-1. Invest Ophthalmol Vis Sci 29:550-557.

Langstan A, Casagrande VA, Fox R (1986) Spatial resolution of the galago. Vision Res 26:791-796.

Lemon V (1986) A monoclonal antibody that binds to cones. Invest Ophthalmol Vis Sci 27:831-836.

Lerea CL, Bunt-Milam AK, Hurley JB (1989) Alpha transducin is present in blue-, green-, and red-sensitive cone photoreceptors in the human retina. Neuron 3:367-376.

Leventhal AG, Ault SJ, Vitek DJ, Shou T (1989) Extrinsic determinants of retinal ganglion cell development in primates. J Comp Neurol 286:170-189.

Marc RE, Sperling HG (1977) Chromatic organization of primate cones. Science 196:454-456.

Martin RD (1989) Primate origins and evolution. London: Chapman Hill.

Muller B, Peichl L, De Grip WJ, Gery I, Korfg HW (1989) Opsin- and S-antigen-like immunoreactions in photoreceptors of the tree shrew retina. Invest Ophthalmol Vis Sci 30:530-535.

Nathans J, Thomas D, Hogness DS (1986) Molecular genetics of human color vision: the gencs encoding blue, green, and red pigments. Science 232:193-202.

Ogden TE (1975) The receptor mosaic of Aotes trivirgatus: distribution of rods and cones. J Comp Neurol 163:193-202.

Packer O, Hendrickson AE, Curcio CA (1989) Photoreceptor topography of the retina in the adult pigtail macaque (Macaca nemestrina). J Comp Neurol 288:165-183.

Pariente $G$ (1979) The role of vision in prosimian behavior. In: The study of prosimian behavior. (Doyle GA, Martin RD, eds), pp 411459. New York: Academic.

Perry VH, Cowey A (1985) The ganglion cell and cone distributions in the monkey's retina: implications for central magnification factors. Vision Res 25:1795-1810.

Polyak S (1953) The vertebrate visual system. (Kluver H, ed) Chicago: University of Chicago.

Rodieck RW (1988) The primate retina. In: Comparative primate biology, Vol 4, Neurosciences (Streklis HD, Erwin J, eds), pp 203278. New York: Liss.

Rohlich P, Szel A, Johnson LV, Kageman GS (1989) Carbohydrate components recognized by the cone-specific monoclonal antibody CSA-1 and by peanut agglutinin are associated with red and greensensitive cone photoreceptors. J Comp Neurol 289:395-400.

Schein SJ (1988) Anatomy of macaque fovea and spatial densities of neurons in foveal representation. J Comp Neurol 269:479-505.

Schnapf JL, Kraft TW, Baylor DA (1987) Spectral sensitivity of human cone photoreceptors. Nature 325:439-441.

Stone J (1981) The wholemount handbook. Australia: Claredon.

Stone J, Johnson E (1981) The topography of primate retina: a study of the human, bushbaby, and New and Old World monkeys. J Comp Neurol 196:205-223.

Szel A, Diamantstein T, Rohlich P (1988) Identification of the bluesensitive cones in the mammalian retina by anti-visual pigment antibody. J Comp Neurol 273:593-602.

Walls GL (1942) The vertebrate eye and its adaptive radiation. Bloomfield Hills, MI: Cranbrook.

Wassle H, Grunert U, Rohrenbeck J, Boycott BB (1989) Cortical magnification factor and the ganglion cell density of the primate retina. Nature 341:643-646.

Wikler KC, Rakic P (1989) Immunocytochemical identification of cones in the retina of diurnal and nocturnal primates. Soc Neurosci Abstr 15:1206.

Wikler KC, Williams RW, Rakic P (1990) Photoreceptor mosaic: number and distribution of rods and cones in the rhesus monkey retina. J Comp Neurol 297:499-508.

Williams RW, Rakic P (1988) Three-dimensional counting: an accurate and direct method to estimate numbers of cells in sectioned material. J Comp Neurol 278:334-352.

Wright PC (1989) The nocturnal primate niche in the New World. J Human Evol 18:635-658. 